\title{
Erratum to: Optical illusion shape texturing using repeated asymmetric patterns
}

\author{
Ming-Te Chi ${ }^{1} \cdot$ Chih-Yuan Yao $^{2} \cdot$ Eugene Zhang $^{3} \cdot$ Tong-Yee Lee $^{4}$
}

Published online: 7 May 2015

(C) Springer-Verlag Berlin Heidelberg 2015

\section{Erratum to: Vis Comput (2014) 30:809-819 \\ DOI 10.1007/s00371-014-0989-3}

In the original article Fig. 5 was published twice, once as Fig. 5 and once again as Fig. 3 by mistake.

The correct Fig. 3 is given below. The publisher apologies for this error.

The online version of the original article can be found under doi:10.1007/s00371-014-0989-3.

\footnotetext{
Chih-Yuan Yao

cyuan.yao@csie.ntust.edu.tw

Ming-Te Chi

mtchi@cs.nccu.edu.tw

Eugene Zhang

zhange@eecs.oregonstate.edu

Tong-Yee Lee

tonylee@mail.ncku.edu.tw

1 National Chengchi University, Taipei, Taiwan, ROC

2 National Taiwan University of Science and Technology, Taipei, Taiwan, ROC

3 Oregon State University, Corvallis, USA

4 National Cheng-Kung University, Tainan, Taiwan, ROC
} 


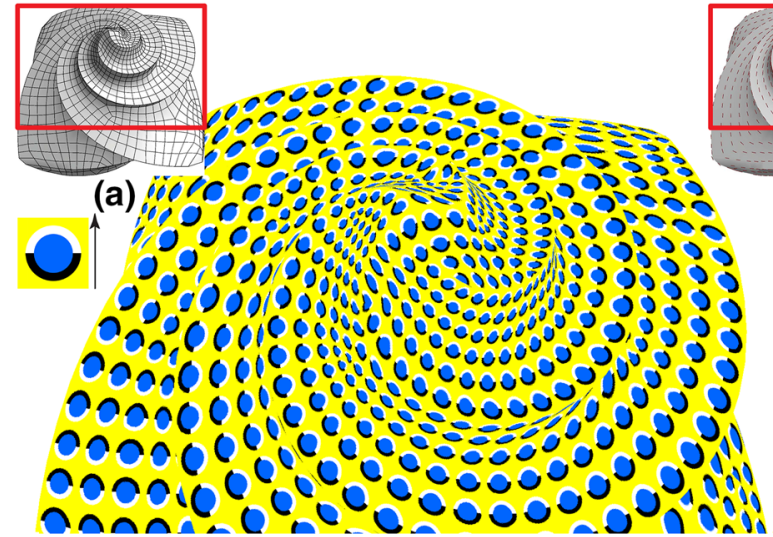

(c)

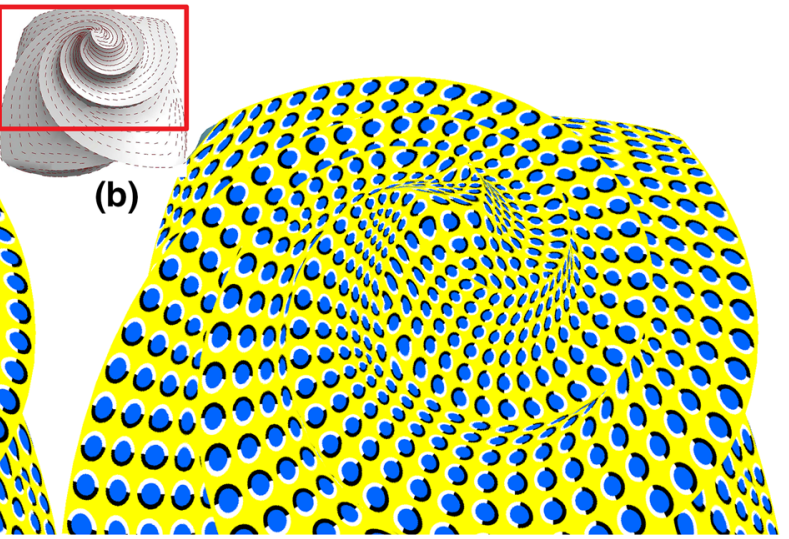

(d)

Fig. 3 An overview of part pipeline overview for shape depiction (without enhancement in background and shading): a quad mesh generation and salient curve extraction, $\mathbf{b}$ curve-guided directional field generation, $\mathbf{c}$ RAP tiling, and $\mathbf{d}$ flow adjustment 